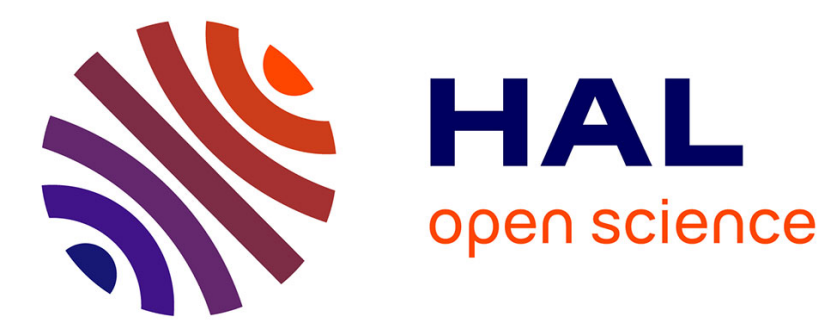

\title{
Photoacoustic visualization of residual stress in ceramic material
}

\author{
R. Burbelo, A. Gulyaev, L. Robur, M. Zhabitenko, B. Atamanenko, Ya. Kryl
}

\section{To cite this version:}

R. Burbelo, A. Gulyaev, L. Robur, M. Zhabitenko, B. Atamanenko, et al.. Photoacoustic visualization of residual stress in ceramic material. Journal de Physique IV Proceedings, 1994, 04 (C7), pp.C7-311C7-314. 10.1051/jp4:1994774 . jpa-00253303

\section{HAL Id: jpa-00253303 https://hal.science/jpa-00253303}

Submitted on 1 Jan 1994

HAL is a multi-disciplinary open access archive for the deposit and dissemination of scientific research documents, whether they are published or not. The documents may come from teaching and research institutions in France or abroad, or from public or private research centers.
L'archive ouverte pluridisciplinaire HAL, est destinée au dépôt et à la diffusion de documents scientifiques de niveau recherche, publiés ou non, émanant des établissements d'enseignement et de recherche français ou étrangers, des laboratoires publics ou privés. 


\title{
Photoacoustic visualization of residual stress in ceramic material
}

\author{
R.M. Burbelo, A.L. Gulyaev, L.I. Robur, M.K. Zhabitenko, B.A. Atamanenko* and Ya.A. Kryl* \\ Physics Faculty, Taras Shevchenko University, 252017 Kiev, Ukraine \\ * Institute for Super Hard Materials, 254153 Kiev, Ukraine
}

\begin{abstract}
The paper presents results of the photoacoustic visualization of fields of residual stress in ceramic material based on silicon nitride. Theoretical analysis yields that the main contribution to the change of photoacoustic (PA) response value is made by nonlinearity of elastic properties of the medium.
\end{abstract}

\section{INTRODUCTION}

Ceramic materials based on silicon nitride are widely used in industry for manufacturing parts of devices working at high temperatures in aggressive process fluid and are subject to considerable mechanical stress. Investigations in this trend aimed both at producing ceramic materials and working out non-destructive methods of control of their properties are continued.

We have made an attempt to use PA microscopy (PAM) for the investigation and monitoring of residual stress field distributions in surface layers of ceramic samples, since it is known that the magnitude of stress influences the defect structure formation and determines physical and mechanical properties. Kasai $M$. and Savada T. [1] demonstrated for the first time the possibility of applying PAM for the visualization of stress fields in an aluminum plate. In ref. [2] the scanning electron acoustic microscope was used to visualize indentation-induced cracks and residual stress in $\mathrm{SiC}$ ceramics.

\section{EXPERIMENT}

Measurements were carried out by means of a computerized PA microscope in which a thermal wave was generated by the chopped $(80 \mathrm{kHz})$ light of an Ar-ion laser and detected using a piezoceramic transducer. The samples were $\mathrm{Si}_{3} \mathrm{~N}_{4}-5 \% \mathrm{Al}_{2} \mathrm{O}_{3}-5 \% \mathrm{Y}_{2} \mathrm{O}_{3}$ composites prepared by sintering or by hot-pressing.

The surface of the samples prepared by sintering were pressed by a Vickers pyramid with a load of $15 \mathrm{~kg}$. The diagonal size of the indentation was about $10-15 \mu \mathrm{m}$. As a result of the Vickers indentations, a stress field was formed which is due to plastic deformation around the indentation. Such stresses can extend to a considerable distance from their formation zone and importantly outside the regions of plastic deformation they can be elastic. The visualization of the field of the residual stresses makes it possible to investigate their distribution and the character of inhomogeneities in the initial material since at inhomogeneities the stress reaches its extrema. Fig. 1 shows the PA amplitude image of the residual stress fields around three 
indentations. One can see that the linear sizes of the region with higher stress values in the case of indentation $2(450 \mu \mathrm{m})$ is considerably larger than in the case of indentation $1(250 \mu \mathrm{m})$ and $3(200 \mu \mathrm{m})$. Note the anisotropic structure of the indentation. All these facts testify the inhomogeneous distribution of physical and mechanical properties of the ceramic material.

For the samples prepared by hot-pressing technology alternations of dark and light regions were observed on the PA images that indicate an inhomogeneous distribution of the PA signal over the surface of the sample. Since extremal conditions of the ceramic synthesis may cause inhomogeneities of structure and phase content, microscale density fluctuations are likely to give rise to deformation fields [3] extending great distances, that exceed essentially the size of inhomogeneities, and result in PA response variations.

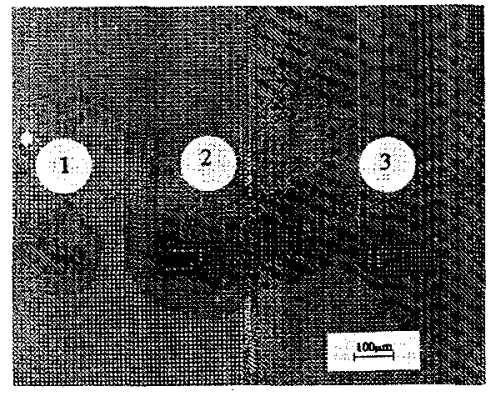

Fig. 1

Analysis of the mechanism of the influence of the residual stress on PA response shows that the modification of elastic properties of a substance under residual stress makes the main contribution to the change of PA signal and this contribution is caused by nonlinearity of elastic properties in the region of elastic deformation.

\section{THEORY}

We calculate the dependence of PA response from the quantity of the residual stress within the scope of nonlinear theory of elasticity. In general, the solution of such a problem is quite complicated, therefore, we use approximations corresponding to the experimental situation.

The model of the system is depicted on Fig. 2.

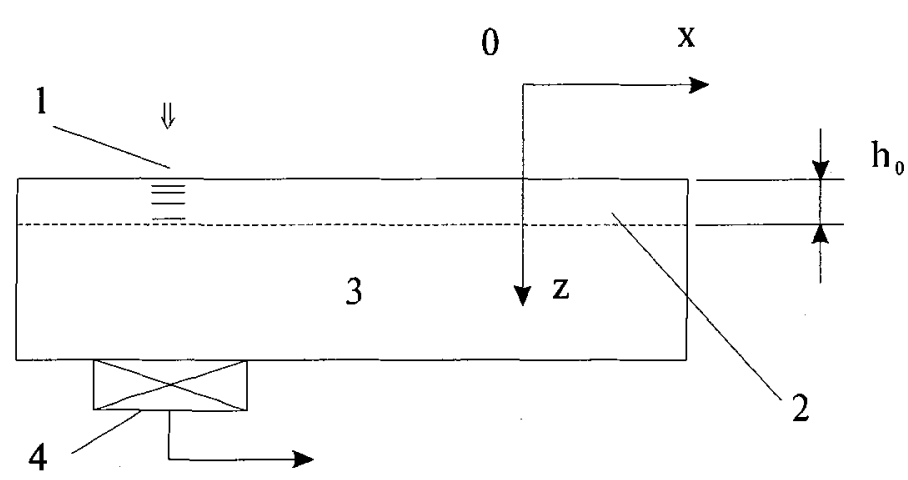

Fig. 2

1: region of incidence of the laser-beam,

2: region of residual stress,

3: volume of the material,

4: piezotransducer of PA signal.

The origin of the coordinates was chosen to be at the surface of the sample. It is considered, that residual stresses are inhomogeneous on the surface, but are averaged in the direction of the $\mathrm{z}$ axis and over layer 
with thickness ho. Therefore, residual stresses can be presented as the following function of coordinates $\mathrm{x}$ and $y$ :

$$
\sigma_{0}=\left\{\begin{array}{rr}
\sigma_{0}(\mathrm{x}, \mathrm{y}) & 0<\mathrm{z}<\mathrm{h}_{\mathrm{o}} \\
0 & \mathrm{z}<\mathrm{h}_{\mathrm{o}}
\end{array}\right.
$$

The geometry of the experiment is such that the PA signal is proportional to the component of elastic displacement in the direction of the $z$ axis. In this case one can consider the task of excitation of the vibrations to be unidimensional and the equations of thermoelasticity can be presented in the following form:

$$
\rho \frac{\partial^{2} U_{z}}{\partial t^{2}}=C^{4} \frac{\partial^{2} U_{z}}{\partial z^{2}}-\gamma \frac{\partial \theta}{\partial z}
$$

where: $U_{z}$ : elastic displacement, $\rho$ : density, $\gamma$ : coefficient of the heat stresses of the material, $C^{+}$: effective elastic constant which is a combination of the elastic constants of the second and third order, and depends on the residual stress, $\theta=\mathrm{T}_{-} \mathrm{T}_{\mathrm{o}}, \mathrm{T}$ : the current value of temperature, $\mathrm{T}_{\mathrm{o}}$ : the initial value temperature. According to ref. [4] it can be shown that in the unidimensional case the effective elastic constant has the following form:

$$
\mathrm{C}^{+}=\mathrm{C}_{11}+\frac{\mathrm{C}_{111}}{\mathrm{C}_{11}} \sigma_{0}(\mathrm{x}, \mathrm{y})
$$

where: $\mathrm{C}_{11}$ and $\mathrm{C}_{111}$ - linear and nonlinear elastic constants.

The temperature in (2) satisfies the equation of heat conduction:

$$
\frac{\partial^{2} \theta}{\partial \mathrm{z}^{2}}-\frac{1}{\mathrm{a}} \frac{\partial \theta}{\partial \mathrm{t}}=\frac{1}{\mathfrak{x}} \mathrm{Q}(\mathrm{q}, \mathrm{t})
$$

where $a$ : thermal diffusivity, $æ$ : thermal conductivity, $\mathrm{Q}(\mathrm{q}, \mathrm{t})$ : power of the heat sources per unit volume. Equation (4) was written assuming that residual stresses do not influence the heat conduction and the distribution of temperature. Under the action of the harmonic light, with modulated power $\mathrm{W}=\mathrm{W}_{0} \mathrm{e}^{\mathrm{j} \omega \mathrm{t}}$, the distribution of temperatures has the following form [5]:

$$
\theta=\frac{W_{0}}{S} \frac{1}{\mathbf{x}} \frac{1}{\left(\frac{\omega}{a}\right)^{1 / 2}} e^{-\left(\frac{\omega}{2 a}\right)^{1 / 2} z} e^{j I \omega t-z\left(\frac{\omega}{2 a}\right)^{1 / 2}-\frac{\pi}{4} J}
$$

assuming light absorption at the surface and neglecting heat losses, where $\mathrm{S}$ denotes the region incidence of the laser beam.

The solution of equation (2) taking into account (5) is too elaborate so let us consider some approximations. The estimations show that the region of thermoelastic stresses for dielectric material which is determined by length $l_{\mathrm{T}}$ on the operational frequency is equal to dozens of micrometers and the length of the acoustic wave $\lambda_{\mathrm{ac}}$ - dozens of centimeters. Thus, the condition $\lambda_{\mathrm{ac}}>l_{\mathrm{T}}$ is satisfied. In this case the thermoelastic excitation of the elastic vibrations is quasistatic and the elastic displacements satisfy the equations for amplitudes:

$$
\mathrm{C}^{+} \frac{\partial^{2} \mathrm{U}_{\mathrm{z}}}{\partial \mathrm{z}^{2}}=\gamma \frac{\partial \theta}{\partial \mathrm{z}}
$$

By integration of equation (6) taking into account that at $T=T_{0}$ the deformations are absent, we obtain: 


$$
\frac{\partial \mathrm{U}_{\mathrm{z}}}{\partial \mathrm{z}}=\frac{\gamma}{\mathrm{C}^{+}} \theta(\mathrm{z})
$$

If we take into account that the distribution of temperature does not depend on $\sigma_{0}(x, y)$ and the photoacoustic signal $\mathbf{P} \sim\left(\partial \mathrm{U}_{z} / \partial \mathrm{z}\right)$ we yield the connection between photoacoustic signal and the value of residual stress:

$$
\mathbf{P}=\frac{\mathrm{P}_{0}}{1+\frac{\mathrm{C}_{111}}{\mathrm{C}_{11}^{2}} \sigma_{0}(\mathrm{x}, \mathrm{y})}=\frac{\mathbf{P}_{0}}{1+\beta}
$$

where $P_{o}$ : a stress-free PA amplitude. For the elastic stress that does not involve distruction of the material, the coefficient $\beta<1$, i.e.:

$$
\mathrm{P}=\mathrm{P}_{0}(1-\beta)=\mathrm{P}_{0}\left[1-\frac{\mathrm{C}_{111}}{\mathrm{C}_{11}^{2}} \sigma_{0}(\mathrm{x}, \mathrm{y})\right]
$$

Thus, as the result of the solution of the thermoelastic equation with approximations corresponding to experimental ones, we have received the dependence of the PA signal on the distribution of the averaged residual elastic stresses on the surface of the sample and the connection of the PA signal with linear and nonlinear elastic constants of the medium.

Taking into account (9) we carry out the estimation of the elastic stresses in the relationship:

$$
\sigma_{0}(x, y)=\frac{C_{11}^{2}}{C_{111}} \frac{P_{0}-P}{P_{0}}
$$

For an arbitrary chosen point on the residual stress field of indentation on Fig. 1: $\frac{P_{0}-P}{P_{0}}=0,025$ and for the elastic constants corresponding to the sample: $C_{11}=3,6 \cdot 10^{11} \mathrm{~Pa}$ and $\mathrm{C}_{111}=67,5 \cdot 10^{11} \mathrm{~Pa}$, we obtain the value of the elastic stresses, which is equal to $\sigma_{0}(x, y) \approx 5 \cdot 10^{8} \mathrm{~Pa}$.

The value of $\sigma_{0}(\mathrm{x}, \mathrm{y})$ that we have obtained, is corresponding to the values reported for the quantities of the elastic stresses in the domain below the limit of strength that testifies to the validity of the proposed mechanism of influence of the residual stresses on the value of the PA signal which is caused by the nonlinearity of the elastic properties of the medium.

\section{REFERENCES.}

[1] Kasai M. and Savada T.,"Non-destructive Evaluation of the Distribution of Stress by Means of the Photoacoustic Microscope", Photoacoustic and Photothermal Phenomena II,(Springer Series in Optical Sciences, Springer-Verlag,62,1990) pp.33-36.

[2] Cantrell J.H., Qian M., Ravichandran M.V. and Knowles K.M., Appl.Phys.Lett. 57 (1990) 18701873.

[3] Sawicka B.D., Murphy J.G., Taheri F., Kanary L.E., Nucl. Instrum. and Meth. Phys. Res. B 69 №2-3 (1992) 365-369.

[4] Physical Acoustics, Ed. by Warren P.Mason (Academic Press, New York and London, 1964,v.1, partA) p.552.

[5] Carslaw H.S., Jaeger J.C., Conduction of heat in solids (Moscow, Nauka, 1964) p.487, (in Russian). 